\title{
Are you serious, Dr Mitchell?
}

\section{Few would have laid money on cells generating energy with proton pumps.}

\section{Leslie E. Orgel}

I he development of molecular biology since its spectacular beginnings in the 1950s has provided the informed participant or onlooker with a series of breakthroughs and surprises. However, few of the insights would have tempted workers in the same field to respond with the question "Are you serious?" In this respect the history of biology is differerent from that of physics, which has included a series of assaults on 'common sense'. It is alleged that distinguished Oxford professors urged their students to ignore relativity theory because it would soon go away. I doubt that many people felt that way about the structure of haemoglobin or DNA. Not since Darwin and Wallace has biology come up with an idea as counterintuitive as those of, say, Einstein, Heisenberg and Schrödinger .

The tradition of molecular biology owes much to the influence of Max Delbruck: formulate precise alternative theories of the phenomenon under discussion, and then design experiments to distinguish between them; but moving too far ahead of the facts is to be discouraged. This approach works wonderfully in biology where, as yet, few surprising general principles have emerged.
One of its consequences, or perhaps one of the consequences of the nature of biology, is that surprising new discoveries are rapidly assimilated. When one first read about reverse transcriptases, introns or ribozymes, one might have wondered about their origins or functions, but one didn't doubt that they were here to stay. The experimental evidence was either already convincing or very soon became convincing.

There may be a few exceptions to this a posteriori transparency of advances in molecular biology but, for personal reasons, one stands out in my memory. In 1955 I took up a position in the Department of Theoretical Chemistry at the University of Cambridge. For the next few years, I worked on problems in inorganic chemistry but, much influenced by George Beadle's elementary course on experimental biology at the California Institute for Technology, I was already spending a good deal of time talking with the Cambridge molecular biologists. One day a young scientist unknown to me made an appointment to talk about a theoretical matter that he thought would interest me. $\mathrm{He}$ was Peter Mitchell, and he wanted to talk about his ideas on how living cells derive energy: his novel chemiosmotic hypothesis (see Nature 191, 144-148; 1961 and, for a

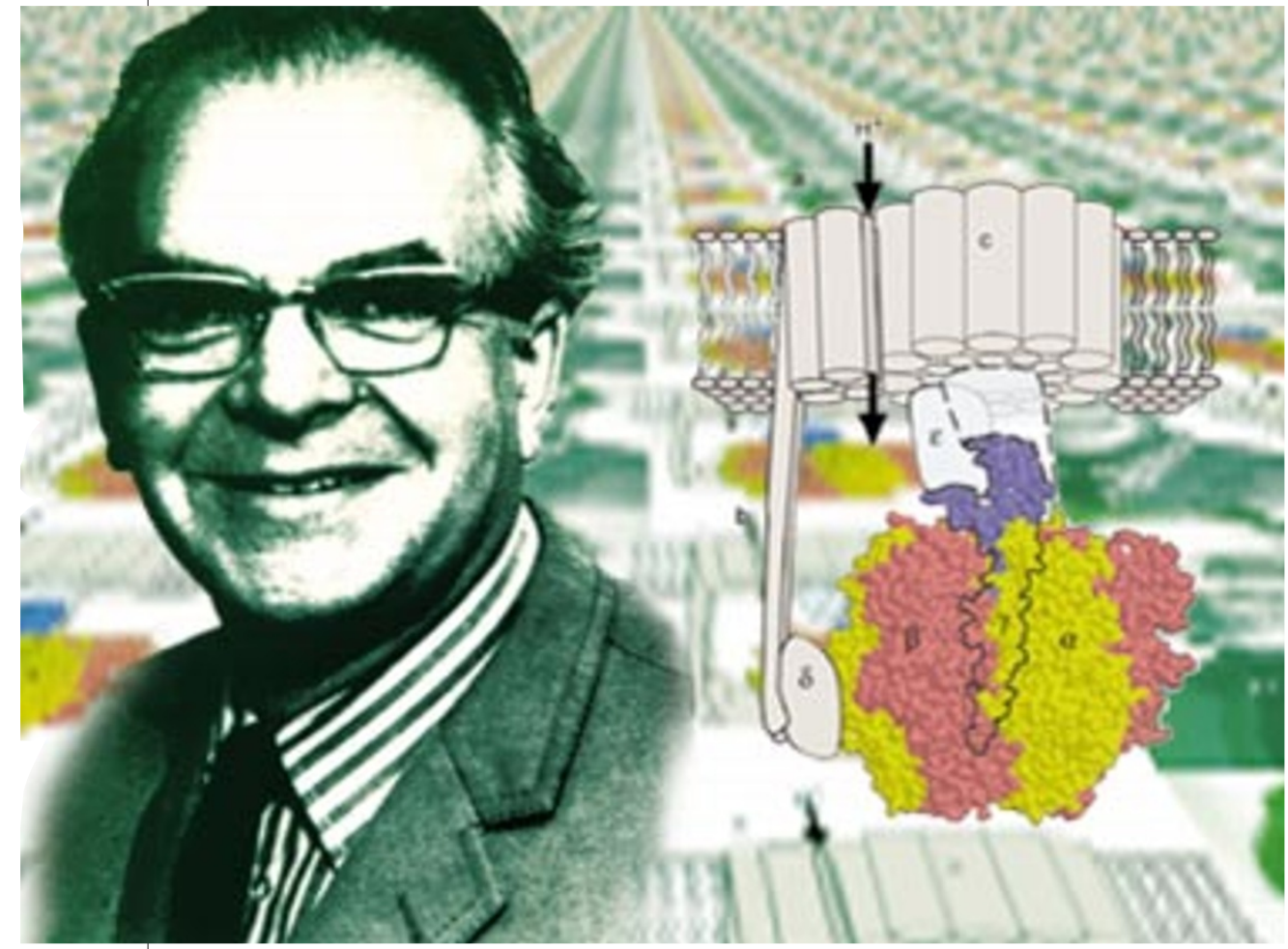

He was serious: Mitchell and ATP synthase, which pumps protons across cell membranes.

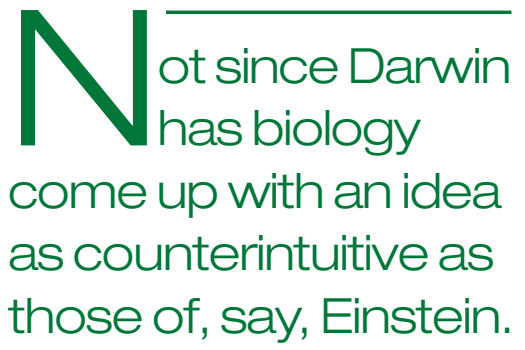

review, Saraste, M. Science 283, 1488-1493; 1999). According to Mitchell's ideas, metabolic energy was used to pump protons across a biological membrane thus establishing a concentration gradient. It was the return of protons down the gradient that led to the synthesis of ATP.

I don't remember the exact date or the details of our conversation, but I do recall its general trend. We discussed in some detail the energy that could, in principle, be obtained by transporting an ion across a membrane from a more concentrated to a less concentrated solution. I don't remember whether we took account of the electrostatic potential. I confirmed Peter's calculation that there might be enough energy available from the transport of protons across biological membranes to generate 'high-energy' phosphate bonds. I was too polite to express a view on the likelihood of Peter's mechanism being correct, but I remember thinking to myself that I would bet anything that ATP synthesis didn't work that way. Fortunately, no one took my bet.

At the time, most people familiar with the problem took it for granted that the formation of ATP would involve the reaction of ADP with a covalent phosphate-containing intermediate. I have no direct knowledge of the attitude of the biochemists, but I know that most molecular biologists shared my scepticism about Peter's ideas. I am not suggesting that his work should be compared to that of Darwin or the great physicists, but it did involve a paradigm shift and his ideas seemed bizarre to most of his contemporaries. They might well have asked "Are you serious, Dr Mitchell?" Of course, he was and he was right. Peter funded himself for much of his subsequent work, so we will never know how his research would have fared at the hands of granting agencies. I wouldn't count on much success in comparable circumstances at the beginning of the next millennium!

Leslie E. Orgel is at the Salk Institute for Biological Studies, 10010 North Torrey Pines Road, La Jolla, California 92037, USA. 